\title{
Optimization of Sparse Randomly Spaced Linear Antenna Array using Hybrid Iteratively Reweighted Least Squares
}

\author{
S. A. Mustapha ${ }^{\mathrm{a}, b, *}$, S. M. Sani ${ }^{\mathrm{a}}$, K. A. Abu-Bilal ${ }^{\mathrm{a}}$ \\ ${ }^{a}$ Department of Electronics and Telecommunication Engineering, Ahmadu Bello University, Zaria, Kaduna \\ State, NIGERIA. \\ ${ }^{b}$ NASENI Solar Energy Limited, Karshi, Abuja, NIGERIA.
}

\begin{abstract}
Uniformly Spaced Antenna Array (USAA) with large radiating elements is characterized with complex feed network as well as high sidelobes level (SLL) leading to interference and power wastage. To solve these problems, research works have been carried out using different methodologies, to synthesize sparse Randomly Spaced Antenna Array (RSAA) to reconstruct the desired radiation pattern using fewer radiating elements and suppressed SLL. In this paper, a deterministic Iteratively Reweighted Least Squares (IRLS) algorithm based on the concept of compressed sensing was used to achieve better sparsity through thinning. The SLL was also suppressed using Convex Technique (CT). The performance of the synthesized array was evaluated in terms of sparsity and SLL. Simulation results showed that it has a higher sparsity of 12 elements with SLL of -39.44dB which are 14.29\% and $28.72 \%$ improvements, respectively compared to previous research work with 14 elements and SLL of -30.64dB.
\end{abstract}

Keywords: uniformly spaced antenna array, randomly spaced antenna array, sidelobes level, mainlobe, iteratively reweighted least squares, sparsity

\section{INTRODUCTION}

An antenna array is a spatial arrangement of two or more radiating elements that are electrically interconnected for the purpose of achieving a desired radiation pattern [1]. In this era of advancement in technologies, there is a compelling need for effective communication especially over long distances where wireless transmission and reception are employed and antenna arrays with large number of elements are always necessary [1]. There are several antenna array configurations in literature some of which are: Linear array, Planar array, Circular array, Cylindrical array, Arbitrary array, and so on [2,3]. In this study, the linear antenna array shown in Fig. 1 was considered because of its simple architecture. These arrays can also be categorized into two, namely: Uniformly Spaced and Non-uniformly (Randomly) Spaced Antenna Arrays [3]. The classical uniformly spaced linear array antennas usually require significantly higher number of elements which can be as large as several thousand $[4,5]$. Hence, sparse Randomly Spaced Antenna Arrays (RSAA) emerged as a solution to feed complexity and high sidelobes level

\footnotetext{
* Corresponding author (Tel: +234 (0)806 165 6986)

Email addresses: sherafding@gmail.com (S. A. Mustapha), smsani_2@yahoo.com (S. M. Sani), kabirahmed@abu.edu.ng (K. A. Abu-Bilal)
}

(SLL) characterized by large Uniformly Spaced Antenna Arrays (USAA) that leads to its prone to interference and power loss using minimum elements [6]. This research work therefore aims at further minimizing antenna elements while suppressing the SLL. Different algorithms have been proposed in the design of these arrays which can be divided into two classes namely: Stochastic and deterministic approaches [8, 9]. Stochastic methods include Genetic Algorithm (GA), Particle Swarm Optimization (PSO), and Simulated Annealing (SA) among others while deterministic approaches include Matrix Pencil Method (MPM), Auxiliary Array Factor (AAF), Almost Different Sets (ADS), Iteratively Reweighted L1 (IRL1) norm minimization, Convex Optimization (CO), Iteratively Reweighted Least Squares (IRLS) and so on $[8,9]$.

For a $2 \mathrm{~N}$ number of antenna elements that are placed symmetrically, the total radiation in the far field for the linear antenna array can be expressed mathematically as $[10,11]$ :

$$
E(\theta)=E_{\text {ele }}(\theta) \times A f(\theta)
$$

where

$$
A f(\theta)=\sum_{n=1}^{N} a_{n} \exp \left[j k x_{n} \sin \theta\right]
$$




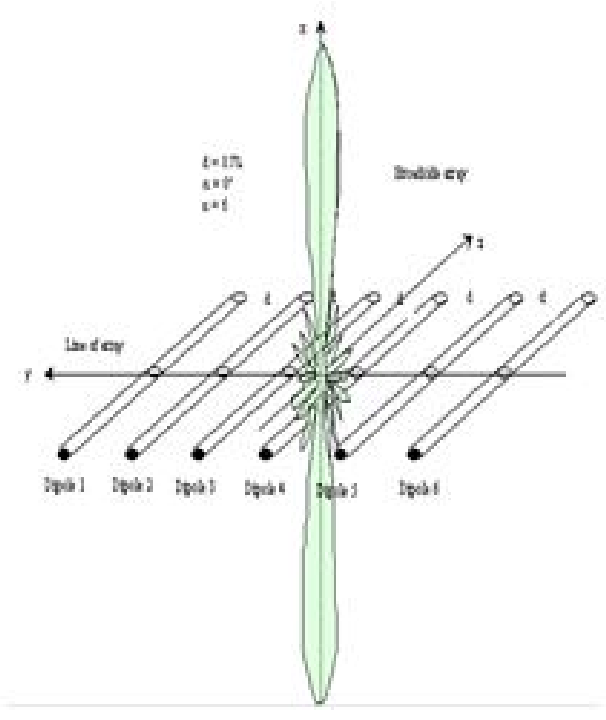

Figure 1: Linear array and radiation pattern of 6 antenna elements [7].

and

$$
x_{n}=\sum_{i=1}^{n} d_{i} \text { for } n \geq 1
$$

where $E_{\text {ele }}$ is the radiation pattern of the individual elements in the array, $A f(\theta)$ is the array factor. $a_{n}$ denotes the excitation amplitude of the $n^{\text {th }}$ element, $N$ is the half of the number of elements in the array, $\theta$ represents the scan angle, $\mathrm{k}$ is the wave number which is equal to $2 \pi / \lambda, \lambda$ is the wavelength, $x_{n}$ is the distance between the first and the last or $n^{t h}$ antenna element, $d_{i}$ is the interelement spacing between the $(i-1)^{t h}$ and $i^{t h}$ elements and $\varphi_{n}$ is the excitation phase shift.

Therefore, Iteratively Reweighted Least Squares was utilized to attain better sparsity. The algorithm was applied based on the concept of Compressed Sensing (CS) to achieve pattern recovery with minimal error. In this research paper, Iteratively Reweighted Least Squares (IRLS) was used. This determined the minimum excitation amplitude which corresponds to the minimum number of antenna elements that reconstructed the desired radiation patter while using convex technique to suppress the SLL. Thereby minimizing the array complexity and improving its performance.

The remainder of the work is organized as follows: section two presents the paper review, section three presents the methodology, section four presents the results and their discussion and section five presents the conclusion.

\section{PAPER REVIEW}

Research works have been carried out in the area of sparsity and SLL improvement in RSLA. Presented below are some literatures highlighting the techniques adopted in reconstructing desired radiation pattern with minimum number of radiating elements.
In a research work, the synthesis of linear sparse arrays of antenna with non-uniform excitation amplitude based on the concept of auxiliary array factor (AAF) to determine the optimal number of array element and their corresponding excitations by mimicking the desired radiation pattern was used without the use of an iterative procedure [12]. The antenna array factor was linearized by discretization after which Fourier Transformation of the array factor was performed. The algorithm achieved minimal computing time. However, the achieved radiation pattern had a higher SLL. In another study, the minimization of the number of antenna elements in a non-uniformly spaced linear array was carried out using a sequential convex optimization technique of weighted l1-norm problem [13]. The optimization problem was transformed into a second order cone programming (SOCP) form by adopting the method of adding slack variables. The technique achieved a reduction in the SLL compared to previous works but failed to achieve sparsity in the number of antenna elements. The application of an iterative convex optimization technique to optimize the positions of antenna elements was carried out for a fixed number of elements in linear and planar arrays with uniform excitation amplitude [14]. This was done in order to achieve a minimized SLL. The array factor (the objective function) was relaxed using Taylor's approximation with a computed slack variable which was pre-determined before transforming the problem to SOCP. The algorithm was able to achieve a radiation pattern with better suppressed SLL when compared with other works. However, the minimization of the number of elements was not considered to achieve sparsity. The optimization of end fire randomly spaced linear antenna array with uniform excitation amplitudes to minimize the SLL in the achieved radiation pattern was investigated using sequential quadratic programming (SQP) algorithm [9]. After comparing the results with several previous works, the research work achieved a reconstruction of the desired radiation pattern with suppressed SLL but with a conspicuous compromise resulting in the shrinking of the beamwidth of the mainlode. The work did not also consider sparsity in the antenna elements. In another work, a hybrid technique for the synthesis of linear array of elements excited with uniform amplitude but varying phases whose radiation pattern has a shaped mainlobe (flat top) was adopted [15]. This was carried out based on linear programming to pre-determine the minimum required antenna array aperture and its corresponding number of elements that will radiate the desired power pattern specifications. An equivalent fast fourier transformation (FFT) was adopted to reshape the array factor before its inverse FFT was carried out. The achieved result showed improvement in SLL. However, due to irregularity in the achieved shape of the mainlobe, the accuracy in the reconstruction could not be guaranteed. An investigation using the extended matrix pencil method (EMPM) 
algorithm to minimize the number of antenna elements required to reconstruct the desired radiation pattern from antenna array was carried out [16]. The properties of both the classical Matrix Pencil Method (MPM) and that of ForwardBackward Matrix Pencil Method (FBMPM) were adopted. The desired radiation pattern functions were first sampled uniformly in the range -1 to +1 based on Nyquist sampling theorem. Then the sampled information was formulated into a composite Hankel Matrix from which the number of elements and the corresponding locations were derived. The reduction in the number of elements was done via singular value decomposition (SDV) of the formulated matrix in order to achieve better approximation. The result from the work achieved sparsity and reduced SLL compared to other previous works but with poor recovery rate due to a significant mismatch at the top of the mainlobe. Further research work studied the use of genetic algorithm (GA) to compute the optimum arrangement of non-uniformly spaced antenna elements for the purpose of suppressing SLL using the inter-element spacing was used as the variable [11]. The result from the work had a better suppressed SLL. However, it was observed that the radiation pattern from the RSLA with the proposed GA optimization algorithm had a larger beamwidth than the benchmark USLA which consequently showed a weak pattern recovery. In a proposal, a hybrid technique to study the maximization of the admissible amplitude excitation tolerances in linear array was carried out [17]. Here, the work was used to compute the difference between inter-element excitation amplitudes to achieve optimal tolerances by applying the interval analysis (IA) based technique after which the particle swarm optimization (PSO) was adopted for the optimization of the amplitudes. The work achieved better sparsity when compared to previous works. However, the research work did not consider randomly spaced linear antenna array thereby limiting the degree of freedom for improvement. Minimizing the SLL below the benchmark technique was also not considered. A two-step optimization based algorithm with the aims of minimizing both the number of elements required to recover the desired radiation pattern and SLL of a randomly spaced linear antenna array using IRL1 minimization algorithm was used [10]. In the proposed algorithm, at each optimization process, reweighted 11 norm minimization was executed, that is, the present excitation amplitudes were obtained from the previous ones to attain solution. The obtained result when compared to several previous works showed an improvement in sparsity and the reduction in the SLL. However, the optimality of the algorithm in terms of sparsity and SLL can still be improved.

It can be seen from the literature reviewed that sparsity and SLL improvement in RSAA are important as they proffer solution to antenna array density, interference and power wastages problems. Many of the previous works did not consider both sparsity and SLL simultaneously while few that considered both required improvement. This paper therefore present an improved sparse randomly spaced linear antenna array using IRLS algorithm to minimize array elements required with suppressed SLL.

\section{METHODOLOGY}

This research work was carried out by first replicating the IRL 1 of [10] before improving on it. This is done by:

1. The following steps were carried out to replicate the existing research work by [10].

i. spreading 1000 elements on $10 \lambda$ aperture length at $0.01 \lambda$ inter-element spacing. Then, the desired mainlobe region parameters were set as: target angle $\theta_{\text {tar }}$ $=0^{\circ}$, array factor with maximum radiation of $A f \theta_{t a r}=1$ i.e., $0 \mathrm{~dB}$ and beamwidth at $-20^{\circ}$ and $20^{\circ}$.

ii. setting the desired peak sidelobes level.

iii. setting threshold value as and the reweighting parameter.

iv. Execution of the IRL1 minimization algorithm using Eq. (4) [10]:

$$
\begin{gathered}
l \min _{x \in \in R^{n}} \sum_{i=1}^{n}\left|w_{i}\right| \frac{1}{\left|w_{i}^{(l)}\right|+\varepsilon} \\
\text { Subject to }\left\{\begin{array}{l}
\left|A f\left(\theta_{i}\right)\right| \leq y \\
A f\left(\theta_{\text {tar }}\right)=1
\end{array}\right.
\end{gathered}
$$

where $\varepsilon$ is a small positive real number used in order to avoid from being undefined, $w_{i}$ is the present excitation weight, $w_{i}^{(l)}$ is the last or previous excitation, $\theta_{t a r}$ is the target angle of the mainlobe, $y$ is the upper bound limit of the sidelobe level, $A f\left(\theta_{i}\right)$ is the array pattern magnitude of the sidelobes region outside the beamwidth of the mainlobe, $\theta_{i}$ represents angles outside the beamwidth, $A f\left(\theta_{t a r}\right)$ is the radiation pattern in the target angle where it attains maximum of $0 \mathrm{~dB}$ when its logarithm is computed.

v. discarding elements with amplitude weighted value below the threshold to achieve sparsity extract the resulta.nt sparse array elements.

vi. Re-optimizing the resultant element excitation weights by minimizing the sidelobes to achieve an effective suppression below the desired sidelobes using Eq. (5) [10]:

$$
\begin{gathered}
\text { minimize } \delta \\
\text { Subject to }\left\{\begin{array}{l}
\left|A f\left(\theta_{i}\right)\right| \leq \delta \\
A f\left(\theta_{\text {tar }}\right)=1 \\
\left|a_{n}\right| \leq 1
\end{array}\right.
\end{gathered}
$$

where $\Delta$ is the half-beam width of the array pattern, $\theta_{t a r}$ is the target angle of 


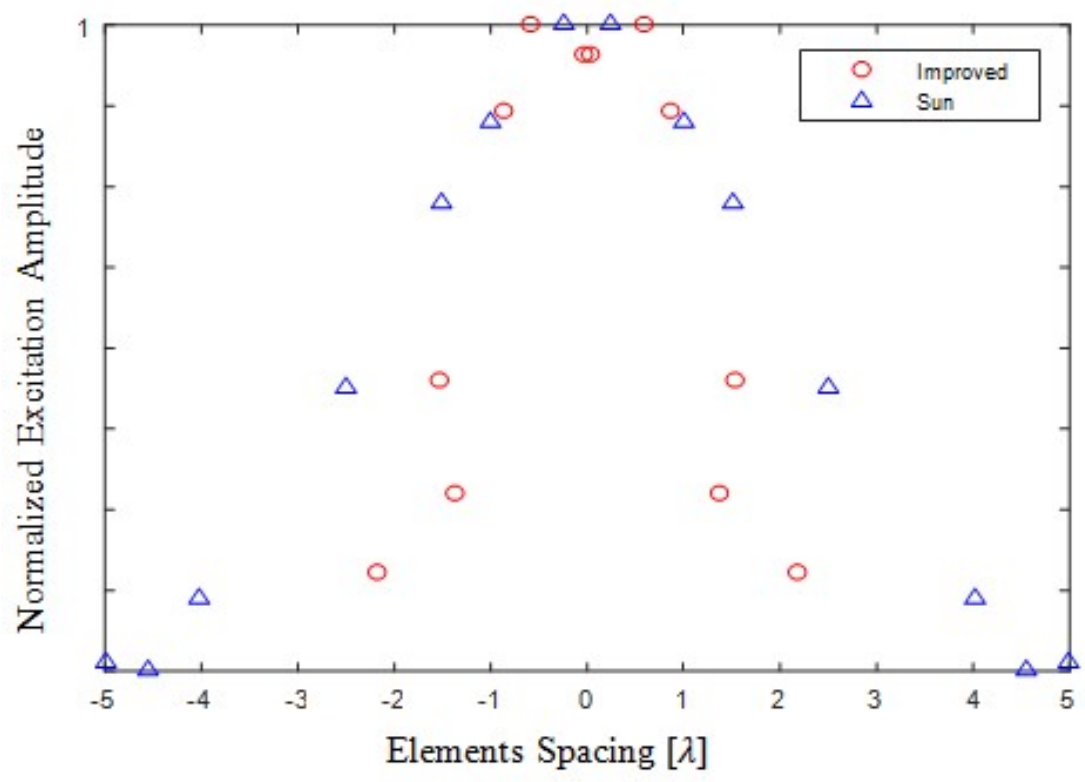

Figure 2: Comparison of achieved sparsity between hybrid IRLS and [10] algorithms.

the mainlobe where the radiation attain maximum magnitude, $A f(\theta)$ is the overall array pattern region, $\delta$ is the upper bound limit of the SLL, $A f\left(\theta_{i}\right)$ is the array pattern magnitude of the sidelobes region outside the beamwidth of the mainlobe, $\theta_{i}$ is the chosen sidelobes angles outside the beamwidth which must satisfy $\left|\theta_{i}-\theta_{\text {tar }}\right|>\triangle, A f\left(\theta_{\text {tar }}\right)$, is the radiation pattern in the target angle where it attains maximum of $0 \mathrm{~dB}$ when its logarithm is computed while $\left|a_{n}\right|$ is the normalized excitation amplitude.

vii. Plotting the sparse randomly spaced linear array radiation pattern

2. In order to improve on the sparsity and sidelobes suppression using IRLS algorithm, the following steps were followed:

i. Steps (i) to (iii) of methodology 1 were repeated except for threshold value set as

ii. Execution of the IRLS algorithm to achieve better sparsity using Eq. (6) [18]:

$$
\begin{gathered}
\min \sum_{i=1}^{n} w_{i}^{2}\left(\left(w_{i}^{(l))}\right)^{2}+\varepsilon\right)^{\frac{p}{2}-1} \\
\text { Subject to }\left\{\begin{array}{l}
\left|A f\left(\theta_{i}\right)_{s l}\right| \leq \delta \\
A f\left(\theta_{\text {tar }}\right)=1
\end{array}\right.
\end{gathered}
$$

where $A$ is the steering vector of the array, $w_{n}$ is the weighting coefficients or the excitation amplitudes whose minimum non-zeros are to be determined, $w_{i}$ is the present excitation amplitude, $w_{i}^{l}$ represents the previous excitation amplitudes, $n=1, \ldots N, N$ denotes the number of antenna elements, $\delta$ is the given upper bound of the SLL for the desired radiation pattern, $A f\left(\theta_{\text {tar }}\right)$ is the target angle pattern of the mainlobe where radiation is maximum and $A f(\theta)_{s l}$ is the entire sidelobe region outside the beamwidth of the mainlobe

iii. iii. Steps (v) to (vii) of methodology 1 were repeated to suppress the SLL.

The result obtained for this work was compared to the research work of [10] in order to validate the achieved improvement using sparsity and SLL as the performance metrics.

\section{RESULTS AND DISCUSSION}

The synthesized antenna array using hybrid IRLS algorithm was simulated and implemented on MATLAB R2015a simulator with the results obtained compared with IRL1 algorithm based on sparsity and SLL. The results obtained for each performance metric are presented thus:

\subsection{Sparsity Achieved by [10] and the Im- proved Algorithm}

The results of the execution of the existing antenna array algorithm by [10] and the IRLS algorithm on the initial large number of antenna elements are presented in this subsection. Initially, 1000 elements were spread over the aperture size as used in the work of [10]. The result of the minimized number of elements with their respective amplitudes obtained to achieve the desired radiation pattern is presented in Fig. 2 . It can be seen from Fig. 2 that the IRLS algorithm used in this research work achieved better sparsity because it was able to use only 12 number of radiating elements to reconstruct the desired radiation pattern as against 14 numbers of elements obtained by IRL1 in the work of [10]. This shows 


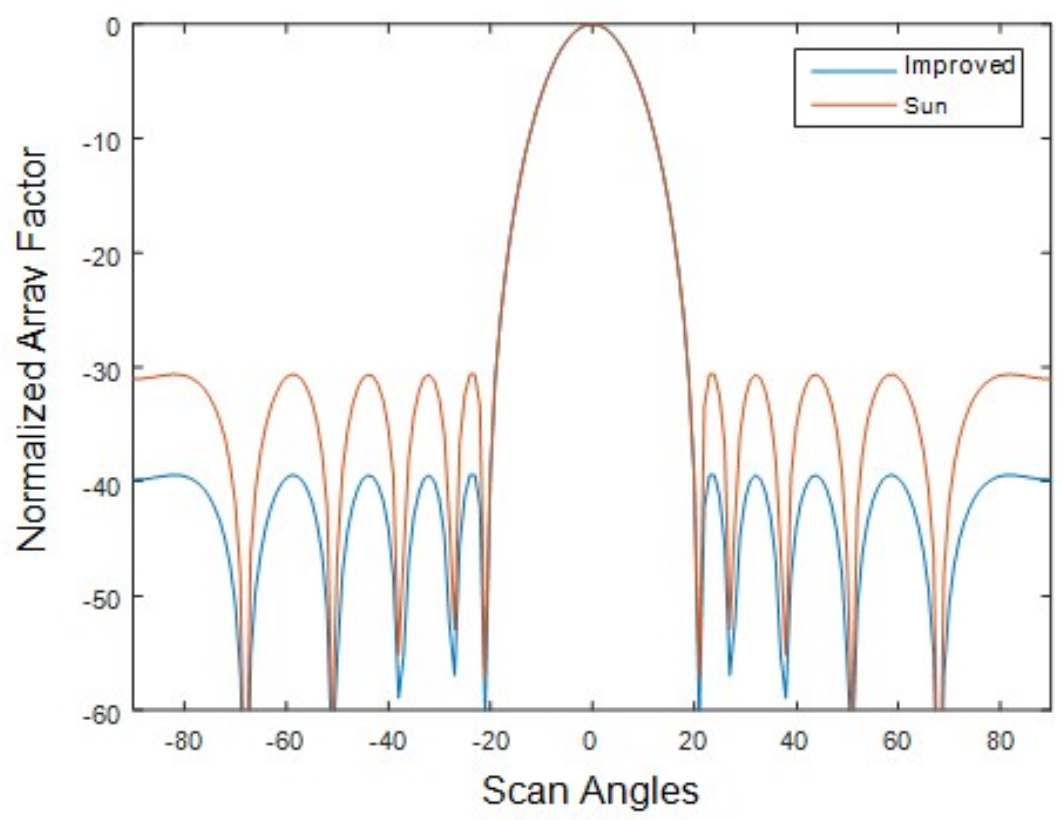

Figure 3: Comparison of achieved SLL Suppression between hybrid IRLS and [10] algorithms.

Table 1: Excitation amplitudes and elements locations.

\begin{tabular}{lcccccccccccccc}
\hline $\begin{array}{l}\text { Excitation } \\
\text { amplitudes }\end{array}$ & 0.038779 & 0.067225 & 0.050542 & 0.107372 & 0.120198 & 0.115883 & 0.115883 & 0.120198 & 0.107372 & 0.050542 & 0.067225 & 0.038779 \\
$\begin{array}{l}\text { Normalized } \\
\text { Excitation } \\
\text { amplitudes }\end{array}$ & 0.322626 & 0.559285 & 0.420489 & 0.893293 & 1 & 0.964101 & 0.964101 & 1 & 0.893293 & 0.420489 & 0.559285 & 0.322626 \\
\hline Location $[\lambda]$ & -2.185 & -1.535 & -1.375 & -0.855 & -0.595 & -0.025 & 0.025 & 0.595 & 0.855 & 1.375 & 1.535 & 2.185 \\
\hline
\end{tabular}

that IRLS algorithm in this work achieved a further reduction in the number of antenna elements with improved sparsity of $14.29 \%$ better than that achieved by IRL1 using Eq. (7) [18]:

$$
\begin{aligned}
& \text { Sparsity }= \\
& \frac{\text { Number of zero - valued array elements }}{\text { Total number of array elements }} \times 100 \%
\end{aligned}
$$

This result shows that there were still some ineffective antenna elements in the sparse array synthesized using IRL1 algorithm by [10]. These ineffective radiating elements have however been successfully discarded in this present research work.

\subsection{Radiation Patterns Achieved after SLL Suppression}

The results of the application of Second Order Cone Programming (SOCP) convex optimization technique for further suppression of the SLL of the achieved radiation pattern from the pattern result from IRLS and that of IRL1 from [10] are presented in Fig. 3.

It can be seen from Fig. 3 that the radiation pattern from the IRLS algorithm used in this research work achieved the desired radiation pattern without compromising the mainlobe charac- teristics as in the work of [10]. Meanwhile, the hybrid IRLS algorithm in this work is seen to have further suppressed the SLL to $-39.44 \mathrm{~dB}$ which is lower than the achieved suppression in the work of [10] which only achieved a maximum SLL of $30.64 \mathrm{~dB}$.

\subsection{Achieved Excitation Amplitudes and Re- spective Elements Locations}

The result of the resultant excitation amplitudes and the respective radiating elements locations of the hybrid IRLS algorithm in this research work are presented in Table 1.

It can be seen from Table 1 that the antenna elements locations are non-uniform but randomly spaced. Also, the elements are symmetrically located such that the locations of half six elements from the 12 elements are a replica of the locations of the other half with a centered zero. This can be verified from Fig. 2.

\section{CONCLUSION}

In this paper, a randomly spaced linear antenna array synthesis technique based on IRLS algorithm and Convex Optimization technique was employed. This research applied the IRLS to minimize the number of antenna elements required to reconstruct the desired radiation pattern in order to have higher sparsity in the array with improved suppression in the SLL. The algorithm is 
Table 2: Excitation amplitudes and elements locations.

\begin{tabular}{llllc}
\hline Synthesis algorithm & $\begin{array}{l}\text { Number of antenna } \\
\text { elements used }\end{array}$ & SLL (dB) & $\begin{array}{l}\text { Percentage SLL } \\
\text { improvement (\%) }\end{array}$ & Sparsity (\%) \\
\hline RSLA [11] & 14 & -30.64 & - & 2.13 \\
RSLA (hybrid IRLS) & 12 & -39.44 & 28.72 & 14.29 \\
\hline
\end{tabular}

applied to the randomly spaced linear antenna array achieved by [10] where 14 elements were used to reconstruct the desired radiation pattern with $-30.64 \mathrm{~dB}$ SLL. The performance of the employed algorithm was evaluated using sparsity and SLL as the metrics. Results from this research work showed that it has a higher sparsity value of only 12 elements which is a $14.29 \%$ improvement, and a SLL of $-39.44 \mathrm{~dB}$ which is a $28.72 \%$ improvement over the existing work of [10]. This therefore means that the density in the elements of such antenna so designed was reduced with a better minimization in loss of transmission power and interference via the SLL. The aim of this research was hereby achieved with an improvement in antenna performance than that achieved by [10] as summarized in Table 2.

\section{References}

[1] J. Carr, Practical antenna handbook, 5th ed. McGrawHill, 2012

[2] G. Lema, G. Tesfamariam, and M. Mohammed, "A novel elliptical-cylindrical antenna array for radar applications," IEEE Transactions on Antennas and Propagation, vol. 64, no. 5, pp. 1681-1688, 2016.

[3] W. Kummer, "Basic array theory," in Proceedings of the IEEE, no. 80, 1992, pp. 127-140.

[4] N. Patel and V. Gupta, "Thinning of linear antenna array: A review," International Journal of Electrical, Electronics and Computer Engineering, pp. 1-4, 2016.

[5] S. Bharti and L. Balai, "Thinning of linear antenna array: A review," International Research Journal of Modernization in Engineering Technology and Science, vol. 2, no. 10, pp. 382-392, 2020.

[6] D. Sartori, L. Manica, G. Oliveri, and A. Massa, "Design of Thinned Arrays with Controlled Sidelobes by ADSCP Strategy," in The 8th European Conference on Antenna and Propagation (EuCAP 2014), IEEE, 2014, pp. $662-665$.

[7] H. Gangadhar, "Radiation pattern for broad side array and end fire array antennas," International Journal of New Technologies in Science and Engineering, vol. 5, no. 5, pp. 97-105, 2018.

[8] G. Prisco and M. D'Urso, "Maximally sparse arrays via sequential convex optimizations," IEEE Antenna and Wireless Propagation Letters, vol. 11, pp. 192-195, 2012.

[9] S. Koziel and X. Yang, "Computational optimization, methods and algorithms," Springer-Verlag Berlin Heidelberg, vol. 11, pp. 1-40, 2011.

[10] B. Sun, S. Xing, J. Zhang, F. Li, Y. Li, and X. Wang, "A novel sparse array synthesis method based on twostep convex optimization," in 2016 10th European Conference on Antennas and Propagation (EuCAP), IEEE, 2016, pp. 1-3.

[11] V. Gangwar, A. Singh, E. Thomas, and S. Singh, "Side lobe level suppression in a thinned linear antenna array using particle swarm optimization," in 2015 International Conference on Applied and Theoretical Computation Technology (iCATccT), IEEE, 2015, pp. 787-790.

[12] D. Caratelli and M. Vigano, "A novel deterministic synthesis technique for constrained sparse array design problems," in IEEE Transactions on Antenna and Propagation, 2011, pp. 4085-4093.

[13] B. Fuchs, "Synthesis of sparse arrays with focused or shaped beam pattern via sequential convex optimiza- tions," IEEE Transactions on Antennas and Propagation, vol. 7, no. 60, pp. 3499-3503, 2012.

[14] B. Fuchs, A. Shrivervik, and R. Mosig, "Synthesis of uniform amplitude focused beam arrays," IEEE Antennas and Wireless Propagation Letters, vol. 11, pp. 11781181,2012

[15] M. Bucci, T. Isernia, and F. Morabito, "An effective deterministic procedure for the synthesis of shaped beams by means of uniform-amplitude linear sparse arrays," IEEE Transactions on Antenna and Propagation, vol. 61, pp. 169-175, 2013.

[16] Y. Liu, Q. Liu, , and Z. Nie, "Reducing the number of elements in multi-pattern linear arrays by the extended matrix pencil method," IEEE Transactions on Antenna and Propagation, vol. 2, no. 62, pp. 652-660, 2014.

[17] N. Anselmi, P. Rocca, M. Salucci, and A. Massa, "Optimization of excitation tolerances for robust beamforming in linear arrays," IET Microwave Antenna Propagation, The Institution of Engineering and technology, pp. $1-7,2016$

[18] Q. Lyu, Z. Lin, Y. She, and C. Zhang, "A comparison of typical lp minimization algorithms," Neurocomputing, pp. 413-424, 2013. 